\begin{tabular}{|c|c|c|c|c|c|c|c|c|c|c|c|c|c|c|}
\hline \multirow{5}{*}{$\begin{array}{l}\text { Observed } \\
\text { symptom }\end{array}$} & \multirow{5}{*}{$\begin{array}{l}\text { Terms } \\
\text { observation }\end{array}$} & \multicolumn{6}{|c|}{ I group. "Grippol plus» $(\mathrm{n}=42)$} & \multicolumn{7}{|c|}{ II group. "Agrippal S1» $(n=37)$} \\
\hline & & \multicolumn{6}{|c|}{ A/California/7/2009/ } & \multicolumn{7}{|c|}{ A/California/7/2009/ } \\
\hline & & \multicolumn{2}{|l|}{ ofH1N1/v } & \multicolumn{2}{|c|}{ A/H3N2/(Victoria) } & \multicolumn{2}{|c|}{ B(Brisbane) } & \multicolumn{2}{|l|}{$\mathrm{H} 1 \mathrm{~N} 1 / \mathrm{v}$} & \multicolumn{2}{|c|}{ A/H3N2/(Victoria) } & \multicolumn{3}{|c|}{ B(Brisbane) } \\
\hline & & Mother & Infant & Mother & Infant & Mother & Infant & Moter & Infant & Mother & Infant & Moter & Infant & \\
\hline & & & & & & & & & & 64,9 & 65,4 & & & \\
\hline & 2-3 day newborn/ & 81,1 & 75,0 & 91,9 & 90,6 & 89,2 & 84,4 & 73,0 & 73,1 & ' & ' & 97,3 & 92,3 & \\
\hline \multicolumn{2}{|l|}{ Seroprotected(\%) } & & 53,1 & & 65,6 & & 59,5 & & 50,0 & 62,2 & & & 73,1 & \\
\hline \multirow[t]{3}{*}{ (?? titer 3 1:20) } & 3 month & 80,0 & $* / \tilde{N}$ & 82,9 & $* / \tilde{N}$ & 88,6 & ${ }^{*} / \tilde{N}$ & 73,0 & ${ }^{*} / \tilde{N}$ & ' & 57,7 & 81,1 & $7^{*}$ & \\
\hline & & & & & & & & & & 35,1 & 34,6 & 2,7 & 7,7 & \\
\hline & 2-3 day newborn/ & 18,9 & 25,0 & 8,1 & 9,4 & 10,8 & 15,6 & 27,0 & 26,9 & $"$ & " & . & $' / \tilde{N}$ & \\
\hline \multicolumn{2}{|l|}{ Seroprotected (\%) } & & 46,9 & 17,1 & 34,4 & & 40,6 & & 50,0 & 37,8 & & 18,9 & 26,9 & \\
\hline \multirow[t]{3}{*}{ (?? titer $<1: 20)$} & 3 month & 20,0 & */N & ** & $* * / \tilde{N}$ & 11,4 & 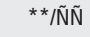 & 27,0 & $* * / \tilde{N}$ & ' & 42,3 & $* *$ & $\gamma^{* *}$ & \\
\hline & & & & & & & & & & & & 91,9 & 80,8 & \\
\hline & 2-3 day newborn/ & 64,8 & 53,1 & 78,4 & 62,5 & 75,7 & 59,4 & 67,6 & 61,5 & 62,2 & 57,7 & $\cdot$ & ' & \\
\hline \multicolumn{2}{|l|}{ Seroprotected (\%) } & & 21,9 & & 12,5 & & 15,6 & & 34,6 & 51,4 & 30,8 & & 42,3 & \\
\hline \multirow[t]{5}{*}{ (?? titer ${ }^{31: 40)}$} & 3 month & 54,3 & $* * / \tilde{N}$ & 71,4 & **/ÑÑ & 74,3 & **/ÑÑ & 62,2 & $* * / \tilde{N}$ & ' & $\eta^{*} / \mathrm{N}$ & 72,9 & $\gamma^{* *} / \tilde{\mathrm{N}}$ & \\
\hline & & & & & & & 31,02 & \pm & & 34,82 & \pm & 85,74 & $\pm 33,64$ & \pm \\
\hline & & 38,19 & $\pm 25,78$ & $\pm 45,95$ & $\pm 31,74$ & $\pm 54,01$ & $\pm 0,19$ & 42,38 & $\pm 37,03$ & $\pm 0,68$ & & 0,28 & 0,5 & \\
\hline & 2-3 day newborn/ & 0,24 & 0,26 & 0,25 & 0,17 & 0,27 & $\tilde{\mathrm{N}}$ & 0,39 & 0,65 & $\cdot$ & $23,78=$ & & $\tilde{N} \tilde{N}$ & \\
\hline & & & 12,59 & \pm & 14,47 & \pm & 13,50 & $\pm 27,32$ & \pm & 23,78 & $\pm 10,91$ & $\pm 47,57$ & $\pm 18,34$ & \pm \\
\hline \multicolumn{2}{|l|}{ Geometric mean } & 30,31 & $\pm 0,22$ & 30,31 & $\pm 0,18$ & 44,89 & $\pm 0,21$ & 0,38 & $20,0 \pm 0,5$ & 550,59 & 0,44 & 0,45 & 0,4 & \\
\hline ( $\log _{2} \mathrm{GMT}$ ??) & 3 month & 0,26 & $* * / \tilde{N}$ & 0,21 & $* * / \tilde{N}$ & 0,23 & $* * / \tilde{N}$ & * & * & * & $* * / \tilde{N}$ & ** & $* * / \tilde{N}$ & \\
\hline
\end{tabular}

seroprotected against $\mathrm{A} / \mathrm{H} 1 \mathrm{~N} 1 / \mathrm{v}$ and $\mathrm{A} / \mathrm{H} 3 \mathrm{~N} 2 /$ accordingly, and 59,4\%, 80,8\% - against influenza B strain (p).

Conclusion When we vaccinate pregnant women against influenza - 53,1-80,8\% infants were seroprotected against vaccine strains of influenza no matter of used vaccine type.

\section{PO-0559 CANDIDA BLOODSTREAM INFECTIONS IN A NICU (2001-2012)}

${ }^{1} \mathrm{~F}$ Pulzer, ${ }^{2} \mathrm{~N}$ Lippmann, ${ }^{1} \mathrm{C}$ Gebauer, ${ }^{1} \mathrm{~A}$ Bläser, ${ }^{1} \mathrm{M}$ Knüpfer, ${ }^{1} \mathrm{U}$ Thome. ${ }^{1}$ Neonatology, University of Leipzig, Leipzig, Germany; ${ }^{2}$ Insitute of Medical Microbiology, University of Leipzig, Leipzig, Germany

\subsection{6/archdischild-2014-307384.1201}

Background and aims Recent reports indicate that the incidence of Candida ssp. bloodstream infection (BSI) is decreasing among NICU patients, and is associated with antifungal prophylaxis and the dwell time of peripherally inserted central catheters (PICCs). This study describes the epidemiology of invasive fungal infections in a single NICU.

Methods We conducted a retrospective study of invasive candidiasis occurring in 6730 infants admitted to our NICU. Positive fungal blood cultures were identified through searching our microbiology database.

Results From a total of 2637 blood cultures performed during the study period, there were $6(<0.1 \%)$ Candida spp. (Candida albicans $\mathrm{n}=5$, Candida lusitaniae $\mathrm{n}=1$ ) BSIs reported. In 3 cases premature rupture of membranes was observed, and in 2 cases maternal vaginal smears were positive for Candida ssp. All Patients with Candida albicans BSI (Gestational age 24.3 to 27.0 weeks) had PICCs, while blood cultures became positive between day 1 to 10.1 patient died on day 13. Treatment consisted in Fluconazole (67\%), Amphotericin B (33\%), and Caspofungin (17\%), partially combined. Persistent positive Candida BSIs were not reported. Systemic antifungal prophylaxis is not implemented at our NICU. During the same period 402 positive fungal cultures in 198 patients were documented from other sources.
Conclusions Even without prophylactic systemic antifungal therapy at our NICU the observed rate of Candida BSIs is low but in line with other studies. Prenatal vaginal smears might help to detect ELBW infants being at high risk for candidemia.

\section{PO-0560 DETERMINATION OF BACTERIAL AGENT IN NEONATAL SEPSIS IN NICU OF IMAM HUSSEIN HOSPITAL OF TEHRAN}

${ }^{1} \underline{Z}$ Rajabi, ${ }^{2} \mathrm{M}$ Soltandallal, ${ }^{2} \mathrm{~S}$ Yaghubi. ${ }^{1}$ Food Microbiology Research Center, Tehran University of Medical Sciences/Medical School, Tehran, Iran; ${ }^{2}$ Pathobilology, Tehran University of Medical Sciences/Medical School, Tehran, Iran

10.1136/archdischild-2014-307384.1202

Background Blood infection is one of the major causes of morbidity and mortality among the newborns especially in developing countries, at the other hand being informed about antimicrobial resistance pattern for correct treatment is necessary. So the aim is study the bacterial agent in blood infection in newborns who were admitted in NICU of Imam Hussein hospital of Tehran and survey their antibiotic resistance pattern.

Methods In this study, microorganisms of blood samples of hospitalised infant in NICU from Imam Hussein, hospital of Tehran during one year isolated, and their antimicrobial resistance pattern was studied by Kirby-Bauer test.

Results During a year study on 105 blood samples, 81(77.1\%) were gram negative and 24(22.9\%) were gram positive organisms. Klebsiella pneumonia (30.5\%), Entrobacter cloacae(21\%) and Staphylococcus epidermidis(11.4\%) were the most common gram negative and positive isolated microorganisms, respectively.

Early and Late onset septicemia in this study occurred with coagulase negative staphylococci and Klebsiella penumoniae. The most antimicrobial susceptibility in gram negative microorganisms were shown to Moxyfloxacin (88.1\%) and Ciprofloxacin $(84.2 \%)$ and in gram positive were shown to Vancomycin $(83.3 \%)$. All the gram positive microorganisms were resistance $(100 \%)$ to penicillin. 
Conclusion This result shown that the most contamination in NICU is from gram negative bacteria.

\section{P0-0561 THE IMPACT OF THE SEPSIS SEVERITY TO THE OXYGENATION OF THE IMMATURE NEONATAL BRAIN}

${ }^{1} \mathrm{D}$ Rallis, ${ }^{2} \mathrm{P}$ Karagianni, ${ }^{2} \mathrm{E}$ Mylona, ${ }^{2} \mathrm{~N}$ Nikolaidis, ${ }^{2} \mathrm{C}$ Tsakalidis. ${ }^{1}$ Paediatrics, Aghia Sophia Children's Hospital, Athens, Greece; 'Neonatal Unit, Papageorgiou General Hospital, Thessaloniki, Greece

\subsection{6/archdischild-2014-307384.1203}

Background and aims Sepsis is major cause of neonatal morbidity and mortality. The level of organ dysfunction is related to the severity of sepsis, as milder episodes seem to lead to less injury. Our aim was to evaluate differences in brain oxygenation, measured by Near Infrared Spectroscopy (NIRS) and estimated via TOI (Total Oxygen Index) and FTOE (Fraction Tissue Oxygen Extraction), according to the sepsis severity.

Methods We designed a prospective study in the 2nd NICU of AUTH, Greece, between 6/2012-12/2012. Neonates with confirmed sepsis underwent 3 NIRS measurements on day 1, 3 and 7 of the episode. Sepsis was classified according to IPSCC criteria into: Sepsis, Severe Sepsis and Septic Shock.

Results Fifty neonates were enrolled with equal birthweight (sepsis: 1610 gr, severe sepsis: 1670 gr, septic shock: 1550 gr) and gestational age $(31,31,30$ weeks respectively).

A TOI decrease/FTOE increase in the 7 th day was recorded in all groups (Sepsis: TOI: 68, 70, 62, FTOE: 29\%, 27\%, 35\%, Severe sepsis: TOI: 66, 70, 62, FTOE: 29\%, 27\%, 33\%, Septic Shock: TOI: 64, 66, 61, FTOE: 33\%, 29\%, 35\%). Neonates with severe sepsis and septic shock required more intensive management and had worst outcome. The brain oxygenation, however, was equally decreased in all 3 groups $(\mathrm{p}>0.05)$, irrespectively to the severity of the septic episode.

Conclusion The outcome of the neonates is proportionally depended to the severity of sepsis, however, even the milder forms seem to cause significant decrease on the brain oxygenation and potential equal brain injury.

\section{P0-0562 PREVALENCE OF GRAM NEGATIVE ORGANISMS ON ROUTINE SURVEILLANCE IN A TERTIARY NEONATAL INTENSIVE CARE UNIT}

${ }^{1}$ MJ Cawsey, 'SV Rasiah, ${ }^{2} \mathrm{~J}$ Gray. 'Neonatal Intensive Care Unit, Birmingham Women's NHS Foundation Trust, Birmingham, UK; ${ }^{2}$ Microbiology, Birmingham Women's NHS Foundation Trust, Birmingham, UK

\subsection{6/archdischild-2014-307384.1204}

Background and aim We perform weekly surveillance for gram negative organisms by rectal swabs and surface swabs for all babies admitted to the neonatal unit. The aim of the audit was to look at the prevalence of colonisation with gram negative bacteria (GNB) and the outcome of these babies.

Methods This was a retrospective review of all positive rectal and surface swabs from $01 / 04 / 13$ to $31 / 03 / 14$.

Results In the last year we had 1465 admissions. The results of the screening programme are shown in the table below. These included GNB on surface or rectal swabs.

Conclusion Preterm babies are at an increased risk of being colonised with GNB at a later date whilst on the neonatal unit. In comparison, term babies were likely to have incidental GNB isolates earlier on. Having a surgical procedure increased the

\begin{tabular}{lll} 
Abstract P0-0562 Table 1 & & \\
\hline & Preterm & Term $>37$ weeks \\
Admissions $n$ & 705 & 760 \\
$n$ with positive swab & $65(9.2 \%)$ & $13(1.7 \%)$ \\
Median day of isolation & $14(0-115)$ & $3(1-40)$ \\
& $28^{+3}$ & $39^{+6}$ \\
Median gest age & $\left(23^{+5}-36^{+5}\right)$ & $\left(37^{+1}-42^{+0}\right)$ \\
& 1060 & 3180 \\
Median birth weight & $(500-3260 \mathrm{~g})$ & $(2280-4820 \mathrm{~g})$ \\
Inborn:Outborn & $57: 8$ & $10: 1$ \\
$n$ had Surgery & $10(15 \%)$ & $2(15 \%)$ \\
Use of Meropenem & $28(43 \%)$ & $1(8 \%)$ \\
Organisms & & \\
Pseudomonas & 13 & 4 \\
ESBL & 10 & 4 \\
Serratia & 17 & 1 \\
Gent resistant organism & 18 & 2 \\
Acinetobacter & 7 & 2 \\
Outcome & & \\
Discharged home & 43 & 9 \\
Transferred out & 15 & 3 \\
Died & 5 & 0 \\
Inpatient & 2 & 1 \\
\hline
\end{tabular}

chances of being colonised with GNB. The use of Meropenem was increased in the preterm population. We routinely isolated these babies with GNB until discharge from the neonatal unit.

\section{PO-0563 DOES EARLY CRP PREDICT CHORIOAMNIONITIS IN VERY LOW BIRTH WEIGHT PRETERM INFANTS?}

${ }^{1}$ E Ryan, ${ }^{2} D$ Eves, ${ }^{2} \mathrm{P}$ Jayadev Menon, ${ }^{2} \mathrm{~S}$ Alnafisee, ${ }^{3} \mathrm{E}$ Mooney, ${ }^{3} \mathrm{P}$ Downey, ${ }^{1} \mathrm{EJ}$ Molloy. ${ }^{1}$ Neonatology, National Maternity Hospital, Dublin, Ireland; ${ }^{2}$ Paediatrics, Royal College of Surgeons, Dublin, Ireland; ${ }^{3}$ Pathology, National Maternity Hospital, Dublin, Ireland

\subsection{6/archdischild-2014-307384.1205}

Background Chorioamnionitis (CA) is associated with preterm birth and adverse neonatal outcomes. The correlation between stage of histological CA, and haematological parameters in the early postnatal period are incompletely defined.

Aim To examine the relationship between CRP and grade of CA in VLBW infants and any associations with neonatal outcomes.

Methods A retrospective review of consecutive infants born $<32$ weeks gestation or $<1.5 \mathrm{~kg}$ at a single centre tertiary referral centre. CA on placental histology was reported as solely maternal inflammatory response (MIR) or both MIR and fetal inflammatory response (FIR). Demographics, haematological parameters and outcomes were recorded.

Results 509 infants were included and histological CA was found in $20 \%$ of placentas but $47.8 \%$ at $<28$ wks. CRP was raised above baseline $(>0.3 \mathrm{mg} / \mathrm{L})$ in $61.8 \%$ of infants with FIR, $64.7 \%$ of infants with MIR and $18.8 \%$ with no CA. CRP on day $1>5 \mathrm{mg} / \mathrm{L}$ was specific for $90.9 \%$ of FIR, 3\% of MIR, and $6.1 \%$ of infants no CA. CRP $>10 \mathrm{mg} / \mathrm{L}$ was $100 \%$ specific for CA but not sensitive.. Advanced FIR on histology (funisitis) correlated significantly with higher CRP. A pathogen was isolated in $0.6 \%$ of CA and blood cultures were positive in $0.02 \%$ of preterm infant with the following outcomes: died $(n=6)$; FIR ( $=8)$; Funisitis $(\mathrm{n}=5)$; High CRP $(\mathrm{n}=5)$. 